\title{
Simulación de Procesos de Negocios (BPSIM) como Soporte Didáctico en el Aprendizaje de la Gestión de Procesos de Servicio
}

\author{
Jaime A. Giraldo y Juanita Pinilla \\ Facultad de Ingeniería y Arquitectura, Universidad Nacional de Colombia Sede Manizales, \\ Carrera 27 \# 64-60, Manizales-Colombia. (e-mail: jaiagiraldog@unal.edu.co, jpinillaa@unal.edu.co)
}

Recibido Abr. 14, 2015; Aceptado Jun. 10, 2015; Versión final Jul. 15, 2015, Publicado Feb. 2016

\begin{abstract}
Resumen
El presente estudio aborda la problemática de cómo mejorar el aprendizaje de procesos de servicios en programas de ingeniería industrial a través de la tecnología Simulación de Procesos de Negocios (BPSIM; Business Process Simulation). Se propone soportar dicho aprendizaje, con enfoque basado en problemas, mediante una herramienta informática que considera el ciclo de modelado de procesos: selección, diagramación, validación, análisis de tiempo, análisis de recursos y análisis de calendarios. La herramienta desarrollada está basada en BPMN 2.0 (Business Process Modeling Notation) y en el uso de un software de libre descarga que incluye un simulador de procesos. El proceso implementado es el bien conocido servicio de contratación e integración de personal en una empresa. Una prueba piloto hecha con estudiantes de ingeniería industrial permite concluir que la herramienta puede llegar a ser de gran utilidad en el aprendizaje de la gestión de procesos de servicio.
\end{abstract}

\section{Business Process Simulation (BPSIM) as a Didactic Support in Learning Management of Service Processes}

\begin{abstract}
This study addresses the issue of how to improve learning of service processes in industrial engineering programs through the technology known as Business Process Simulation (BPSIM). It is proposed to support such learning, based on the problem solving methodology employing a software tool that considers the cycle process modeling approach: selection, layout, validation, time analysis, resource analysis and analysis schedules. The tool developed is based on BPMN 2.0 (Business Process Modeling Notation) and in the use of an open-access software which includes a process simulator. The process implemented is the well-known service recruitment and integration of staff in a company. A pilot test done with a group of industrial engineering students allows concluding that the tool can be useful in the learning of process management service.
\end{abstract}




\section{INTRODUCCIÓN}

El ritmo de vida acelerado en el que se está inmerso en esta era globalizada y soportada en Tics, trae consigo para los nuevos profesionales la necesidad de adaptarse constantemente a las nuevas exigencias del medio con el fin de mantenerse competitivo. Si partimos de que la educación, según Suarez (1985), tiene como fin "conocer el mundo lo suficiente para poder enfrentarlo con eficiencia", Ventura (2011) considera que debe entonces re-conceptualizarse el entorno universitario para promover el pensamiento crítico-reflexivo requerido bajo los nuevos paradigmas sociales. Para Vives (2010), el aprendizaje deja de ser entendido como un cambio neto de conducta para considerarlo el resultado de la interiorización de un objeto, más por descubrimiento que por recepción de saberes y del mismo modo, la enseñanza entendida como la transmisión de conocimientos se enriquece y se torna en un proceso dinamizador de ese aprendizaje.

Para Riascos et al. (2009), las Tics como mediadoras en el aula de clase contribuyen a la construcción del conocimiento, tal como se plantea en la estrategia ABP (Aprendizaje Basado en Problemas) e invitan al estudiante al aprendizaje autónomo, donde el docente deja de ser un transmisor y se vuelve un acompañante y guía en la asimilación del conocimiento. De este modo, el cambio de paradigma conlleva al desarrollo de nuevas metodologías y competencias en los participantes del proceso educativo. De acuerdo al estudio realizado por estos autores, los docentes consideran que estos recursos tecnológicos permiten el mejoramiento de la labor de enseñanza, pues los enriquecen brindándoles la oportunidad de acceder a la información de manera fácil, y acercándolos a los estudiantes. De igual manera, Casadei et al. (2008), afirman que las Tics refuerzan el interés de los estudiantes por el aprendizaje. Como se plantea anteriormente, las herramientas tecnológicas potencian la enseñanza - aprendizaje.

Para Marquez et al. (2011) y Morales (2008), al hablar de un aprendizaje por descubrimiento se hace referencia a la estrategia del aprendizaje basado en problemas (ABP), metodología constructivista y didáctica que se aplica hace más de cuarenta años en los centros de enseñanza, y que pretende que el estudiante resuelva un desafío que se le presenta de manera autónoma y creativa, mediante la búsqueda de la información que él crea pertinente. El desarrollo de esta estrategia requiere que exista compromiso y motivación personal del educando para construir escenarios de trabajo en equipo y comunicación asertiva, en donde pueda darse la exploración de alternativas en su búsqueda del conocimiento.

En el contexto de los procesos de negocios, Barjis y Verbraeck (2008), consideran que una empresa promedio en un ambiente global podría verse como una red de cientos de procesos de negocio entrelazados, señalando de ejemplo a SAP (ERP ampliamente usado), el cual consiste de mínimo 604 procesos empresariales debidamente encadenados. De otra parte, para González et al. (2014), una de las áreas de trabajo más importantes de la ingeniería industrial la constituye la comprensión y modelamiento de procesos empresariales, que pueden ser de manufactura soportada por servicios de apoyo o puramente de servicios; no obstante, tradicionalmente la metodología empleada ha hecho mayor énfasis en los procesos manufactureros, dejando de lado los procesos administrativos y de servicios, que cada vez tienen mayor relevancia y participación en el mercado globalizado.

Como medio para la comprensión de los procesos aparece la modelación. Para Bolaños y López (2014), el modelado de procesos organiza la información correspondiente a la estructura y funcionamiento de un sistema, tal como sus entradas, los procesamientos/controles que se realizan y las salidas, y permite visualizar los flujos de trabajo dentro de los procesos, así como la interacción entre todos los entes que intervienen en él. De acuerdo a los atributos que se desee expresar en el modelo y al grado de detalle con que quiera representarse el sistema, existen diversas notaciones para modelar los procesos; entre ellos se destacan los diagramas de flujo de datos (DFD), Event-driven Process Chain (EPC) y Business Process Modeling Notation (BPMN). Según Espinoza y López (2013), esta última notación hace parte de un nuevo paradigma para la aplicación de las tecnologías de la información y comunicación en las organizaciones, denominada genéricamente Business Process Management (BPM), que incluye un conjunto de herramientas para mejorar la eficiencia/efectividad del negocio y alcanzar una ventaja competitiva. La implementación de BPM en las empresas está soportada por la tecnología BPMS (Business Process Management Systems), software especializado para el análisis de procesos de negocio, su modelamiento y posterior automatización y constante mejora.

Las fases sobre las que operan los BPMS consisten principalmente en el modelamiento o diseño, la automatización (incluyendo interacción con sistemas ERP y CRM) y la identificación de oportunidades de mejora de los procesos en su implementación. La etapa de diagramación (la cual se realiza mediante BPMN) es crucial para definir, analizar y comprender el proceso, pues permite representar de manera estructurada el flujo de trabajo a lo largo de él, así como los participantes y las reglas de negocio (Espinoza y López, 2013). Para Bolaños y López (2014), BPMN ha alcanzado alta popularidad por ser una notación 
sencilla y clara para todos los miembros de la organización, desde los analistas hasta los operadores, facilitando así reducir la brecha o gap entre el diseño de nuevos procesos y su automatización y posterior implementación; muchos proveedores importantes de Tics emplean esta notación como estándar, la cual es impulsada y especificada por OMG (Object Managament Group) en su sitio www.omg.org.

De otra parte, las tecnologías de simulación, permiten representar una realidad específica mediante un modelo lógico-matemático. A través de esta tecnología, el estudiante se ubica en un contexto en el cual se vuelve un participante activo del proceso, pues puede interactuar con el modelo y explorar los efectos de sus decisiones en el funcionamiento del mismo (Giraldo et al, 2013). Esta interacción consiste en cambiar parámetros de operación (lógicos o matemáticos) del modelo y analizar los resultados obtenidos luego de las modificaciones para entonces proponer alternativas de mejoramiento.

Aparece recientemente entonces una tecnología que integra la modelación de procesos (BPM) con la simulación. Business Process Simulación, o mejor conocido por el nombre informático que permite aplicarlo, BPSim, la cual consiste en el empleo de tecnologías de simulación de los procesos de negocio, con el objetivo de efectuar mejoras antes de automatizarlo e implementarlo (Melao y Pidd, 2003). Según el estudio realizado por estos autores (en los albores de esta nueva tecnología) sobre los campos en los que se ha empleado BPSim, aproximadamente el $43 \%$ de los usuarios de la herramienta pertenece a los sectores público y de servicios, este último de gran relevancia en el presente análisis, mientras el $18 \%$ es de la industria manufacturera y de distribución y el $39 \%$ corresponde a otros sectores. De acuerdo a estos mismos autores, para quienes utilizan BPSim, el software les permite experimentar con los procesos, así como comprender de manera profunda las interacciones de los participantes en los procesos más complejos.

De hecho, en IBM (2015) se describe una herramienta informática dirigida a profesionales de TI y de negocios (por ejemplo ingenieros industriales) que utiliza simulación y BPM con el objetivo de lograr que ambas perspectivas profesionales entiendan que los procesos son críticos para el éxito empresarial, para lo cual se plantean modelos que representan escenarios sobre procesos de tráfico vehicular, centro de llamadas (call center) y cadenas de abastecimiento. En Oracle (2015), mencionan que el producto de software denominado Oracle BPM, permite correr simulaciones, mediante las cuales analistas de negocios y desarrolladores $\mathrm{TI}$ puedan predecir el comportamiento de procesos de negocios bajo condiciones específicas, pudiendo verificar que la salida satisface las métricas establecidas en los objetivos e identificar cuellos de botella e igualmente pueden simular para probar los efectos de cambios sobre el diseño de un proceso existente. En Bizagi (2014), describen las potentes capacidades de simulación de su modelador BPMN que permite a sus usuarios tomar mejores decisiones mediante la visualización del impacto de cambios propuestos a los procesos empresariales antes de su implementación en el mundo real.

A partir de los planteamientos anteriores, se quiere divulgar el desarrollo de una herramienta informática empleando la tecnología BPSim, mediante la cual se facilite el aprendizaje (empleando un enfoque ABP) de la gestión de procesos administrativos y de servicios a estudiantes de ingeniería industrial y afines, utilizando como ejemplo el conocido proceso de contratación e integración de personal en una empresa. Inicialmente la herramienta permite al docente explicar a través de un diagrama escrito en BPMN los pasos del proceso y posteriormente, a través de simulación, facilitar a los estudiantes evaluar diferentes alternativas de mejora del proceso en términos de uso de recursos y tiempos de respuesta.

\section{DESARROLLO METODOLÓGICO}

La Fig. 1 representa las fases de la metodología seguida en la elaboración de la herramienta BPSim.

\section{Selección del proceso/problemas}

A partir de los conocimientos adquiridos en el área de gestión humana en las organizaciones, se planteó un modelo del proceso de contratación e integración de personal a una compañía, de modo que la herramienta no sólo fuera útil en la ilustración de la dinámica de los procesos de servicio, sino que además funcionara como medio para representar un proceso de apoyo cotidiano para cualquier empresa. Igualmente se propone para representar diversas variantes de problemas asociados al proceso seleccionado, las siguientes dimensiones con base en los aportes dados por (Vizcarro y Juarez, 2008) en el contexto de ABP: Enunciado del problema; Relación con el conocimiento previo de los discentes; Elementos desconocidos del problema que demanden más información; Interés y relevancia para los estudiantes; Objetivos de aprendizaje formulados por los estudiantes sobre la(s) materia(s) deseada(s) y Reflejar la complejidad de los problemas de la vida real. 


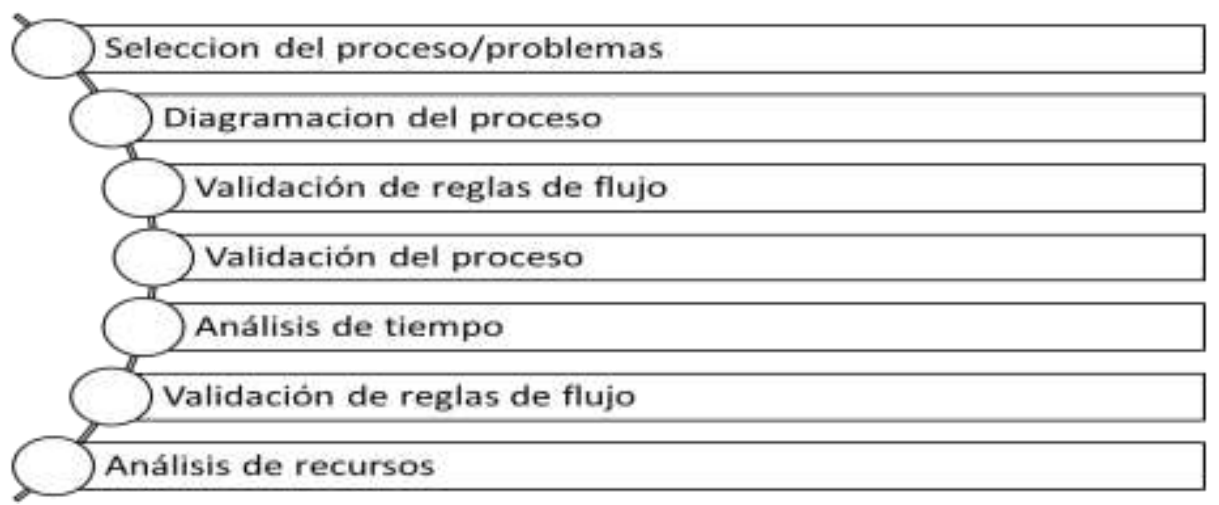

Fig. 1: Metodología seguida en la elaboración de un modelo de simulación mediante BPSim (Datos de Bizagi (2014)

\section{Diagramación del proceso}

El diagrama del proceso se elaboró mediante el diagramador de procesos de libre descarga Bizagi Modeler (Bizagi, 2014), el cual sigue el estándar BPMN 2.0. Este proceso consiste básicamente en la preparación de la documentación legal necesaria para firmar el contrato, y la disposición de los elementos del puesto de trabajo que ocupará el nuevo empleado. Se reconocen dos fases en el proceso: la fase previa al ingreso del empleado y la primera semana de labor, luego de la firma del contrato. Los participantes en las actividades son el nuevo empleado, el área de talento humano, el jefe del nuevo empleado y el administrador del área de tecnología. Nótese que los participantes, a excepción del nuevo empleado, son internos de la organización.

En la figura 2 se expone una vista parcial del diagrama del proceso, en la cual se muestra la secuencia de actividades a partir del evento (selección de un empleado) que inicia una contratación e integración de un empleado (instancia de proceso). Mediante este diagrama se espera que el docente explique el proceso a los estudiantes desde dos perspectivas: a) Estática: mostrando la secuencia en que se ejecutan las tareas, eventos, decisiones, etc., incluyendo para cada símbolo una ayuda en línea y b) Dinámica: simulando un numero determinado de instancias del proceso. Para ver el diagrama completo y un manual de ayuda para usarlo y simularlo acceder al sitio www.bpsimservicios.wordpress.com.

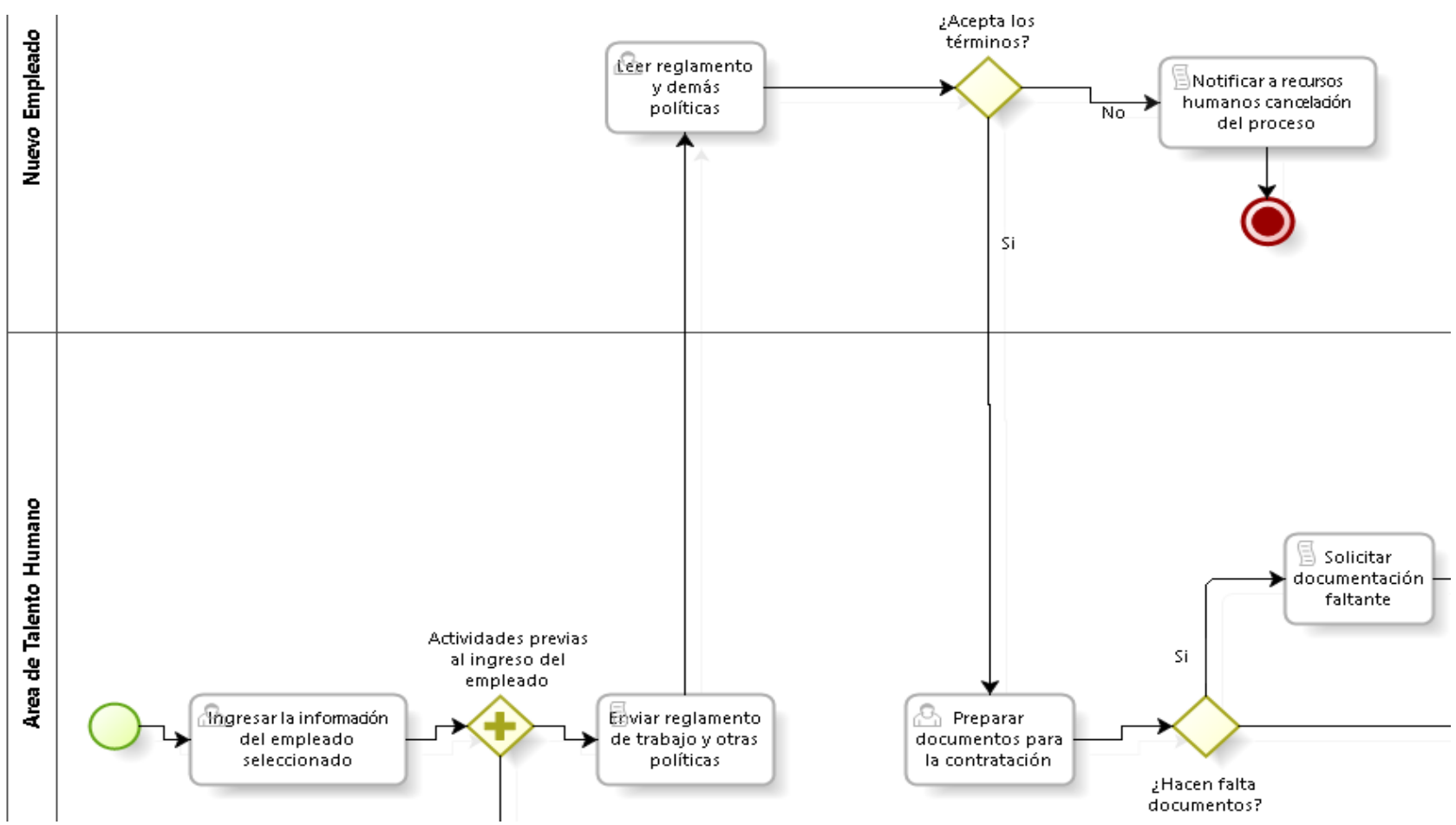

Fig. 2: Representación parcial diagrama del proceso de contratación e integración de personal en formato BPMN 2.0. Fuente: Adaptación propia a partir de plantilla proporcionada en www.bizagi.com. 


\section{Validación de reglas de flujo}

Luego de la diagramación, se procedió a validar el modelo según las reglas de decisión del proceso, que radican en si el nuevo empleado está de acuerdo con los términos propuestos en las políticas y demás normativa de la compañía (Véase Figura 3) y si los documentos necesarios para llevar a cabo el proceso de contratación fueron recibidos en su totalidad por la empresa (Véase Figura 4), entre otras.

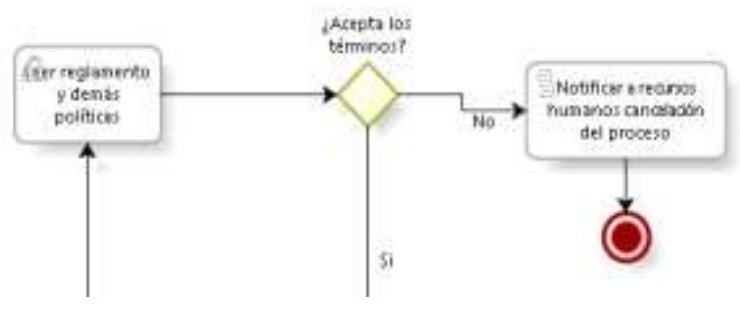

Fig. 3: Validación reglas de flujo de proceso tras la lectura de la normativa.

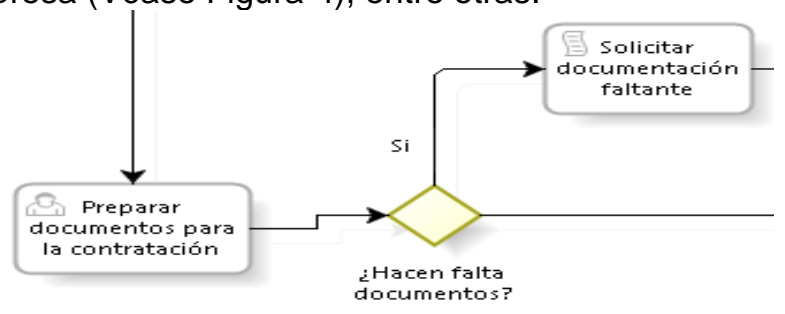

Fig. 4: Validación reglas de flujo de proceso tras la preparación de la documentación.

\section{Validación del proceso}

En el primer paso de la simulación se programan las compuertas de decisión con las probabilidades correspondientes a cada uno de los flujos de proceso. Así por ejemplo, la Figura 5 muestra como la compuerta ¿Acepta los términos? tiene un $95 \%$ de que le flujo de trabajo vaya por el "Si" y el $5 \%$ por el "No". En este punto, el modelo se ejecutó para verificar su validez.

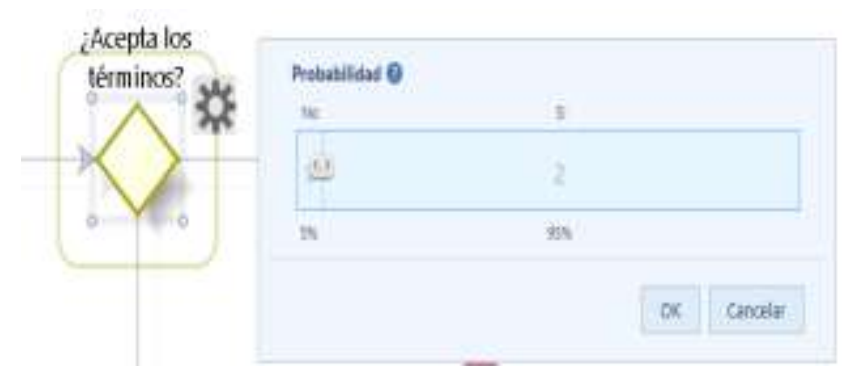

Fig. 5: Paso 1 de la simulación, validación del proceso a través de compuertas divergentes.

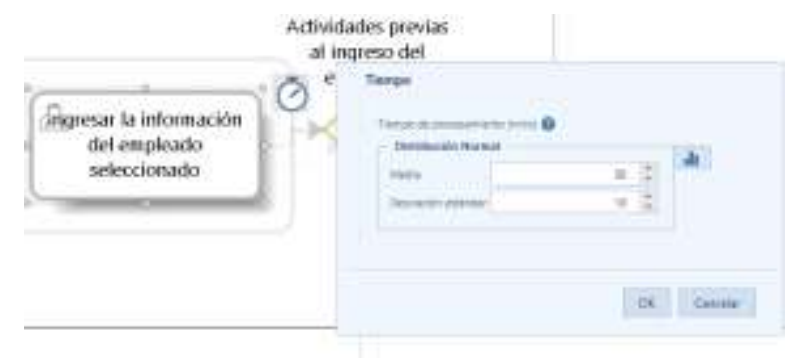

Fig. 6: Paso 2 de la simulación, asignación de tiempos de duración de las tareas.

\section{Análisis de tiempo}

En el segundo paso, análisis de tiempo, se establece la duración de cada tarea, y teniendo en cuenta su naturaleza se asignan valores determinísticos o probabilísticos a los tiempos. Así por ejemplo, la Figura 6 muestra que el tiempo de la tarea "Ingresar la información del empleado seleccionado" se distribuye probabilísticamente mediante una distribución normal. También en este paso se programa el funcionamiento de los eventos temporizados.

\section{Análisis de recursos}

En el análisis de recursos, que es el tercer paso de la simulación, se asignan los recursos necesarios para la ejecución de cada una de las tareas. Así por ejemplo, la Figura 7 muestra que la tarea "Enviar el reglamento de trabajo y otras políticas" es realizada por el recurso "Analista de talento humano".

\section{Análisis de calendarios}

En este paso se establecen los turnos laborales de la compañía en que se desarrolla el proceso y el periodo del año que la empresa labora. La Figura 8 muestra que el "Turno de la mañana" comienza a las 7:30 am, dura 4 horas y se repite diariamente.

\section{Informe de uso de recursos y tiempo de proceso}

Mediante este informe se espera satisfacer necesidades de información sobre los recursos empleados en el proceso (punto de vista del administrador) y necesidades de información sobre los tiempos de respuesta del proceso (punto de vista de los clientes del proceso), a fin de lograr que los estudiantes propongan mejoras en el desempeño de este. 


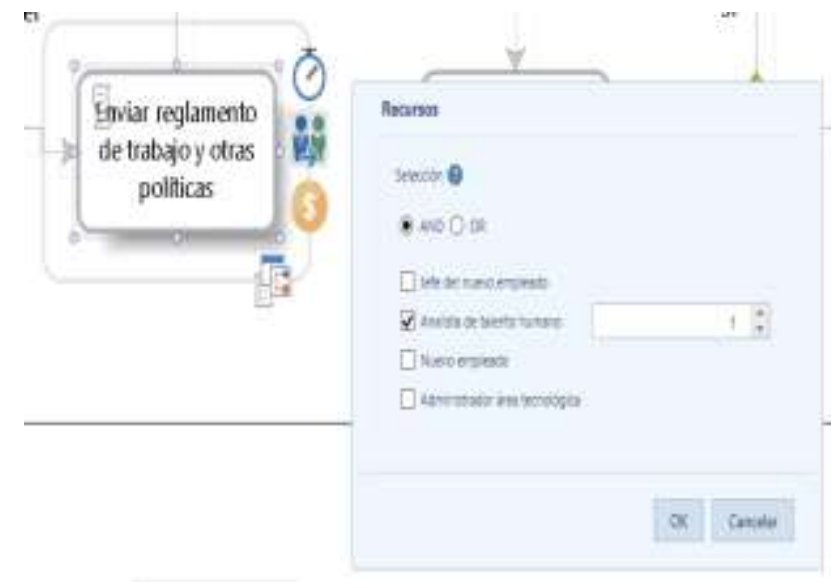

Fig. 7: Paso 3 de la simulación, asignación de recursos a las tareas.

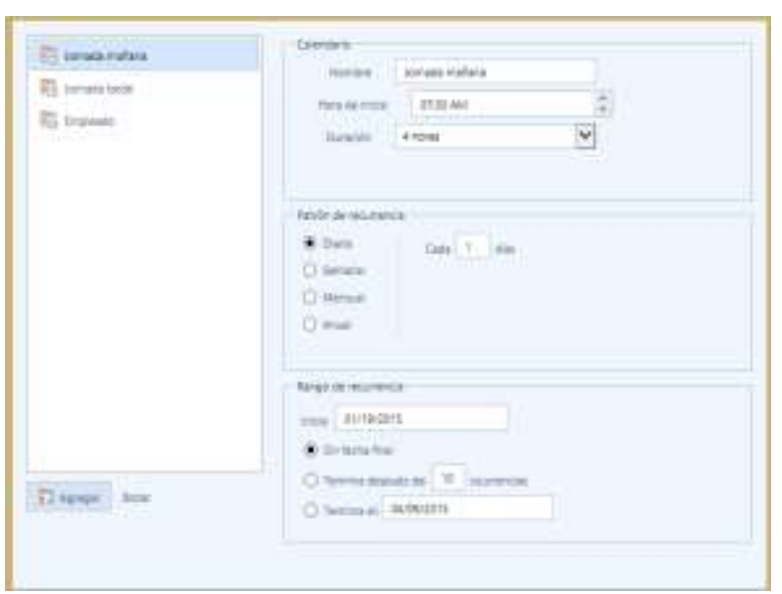

Fig. 8: Paso 4 de la simulación, creación de calendarios.

\section{RESULTADOS Y DISCUSION}

Se espera que los estudiantes tengan claro que el objetivo de la diagramación del proceso en BPMN es su posterior automatización e implementación mediante un BPMS. Es por esto que a fin de garantizar un desempeño eficiente (uso de recursos) y efectivo (logro de los resultados) del proceso, antes de su implementación, se sugiere que los discentes simulen el proceso, tomando por su propia cuenta algunas decisiones relacionadas con: número de instancias de proceso a evaluar, tiempos estimados por tarea, probabilidad por flujo de trabajo, cantidad de recursos a utilizar y horarios de trabajo; es decir, aplique la metodología propuesta en el inciso anterior. Se describe a continuación los resultados básicos obtenidos al simular 100 contrataciones de empleado, teniendo en cuenta la descripción del problema a solucionar en el contexto ABP, según se muestra en la Tabla 1. Los valores de decisión (recursos, tiempos, etc.) detallados utilizados en la simulación pueden ser consultados en el sitio www.bpsimservicios.wordpress.com.

Tabla 1: Descripción del problema a solucionar

\begin{tabular}{|l|l|}
\hline $\begin{array}{l}\text { Enunciado del } \\
\text { problema }\end{array}$ & $\begin{array}{l}\text { ¿Cuáles han de ser los valores aproximados de los tiempos de las tareas que permitan } \\
\text { realizar el proceso en no más de } 20 \text { días al contratar e integrar 100 nuevos } \\
\text { empleados? }\end{array}$ \\
\hline $\begin{array}{l}\text { Relación con el } \\
\text { conocimiento previo } \\
\text { de los discentes }\end{array}$ & El discente debe haber aprobado un curso básico de gestión empresarial \\
\hline $\begin{array}{l}\text { Elementos } \\
\text { desconocidos del } \\
\text { problema que } \\
\text { demanden más } \\
\text { información }\end{array}$ & $\begin{array}{l}\text { Debería el estudiante indagar por: comportamiento de la demanda de servicios de } \\
\text { contratación e integración de personal, tiempos de duración de tareas, expresándolos } \\
\text { en términos de distribuciones de probabilidad. Además preguntarse por la duración de } \\
\text { la jornada laboral. }\end{array}$ \\
\hline $\begin{array}{l}\text { Interés y relevancia } \\
\text { para los estudiantes }\end{array}$ & $\begin{array}{l}\text { El interés se da en razón a poder experimentar con un sistema virtual, casi a costo cero. } \\
\text { La relevancia reside en trabajar con objetos de estudio propios de la ingeniería industrial } \\
\text { (ambiente empresarial) }\end{array}$ \\
\hline $\begin{array}{l}\text { Objetivos de } \\
\text { aprendizaje } \\
\text { formulados por los } \\
\text { estudiantes sobre } \\
\text { la(s) materia(s) } \\
\text { deseada(s) }\end{array}$ & $\begin{array}{l}\text { Conceptos sobre demanda de servicio; estructura (recursos, operaciones) de tareas } \\
\text { para prestar un servicio; secuencia de las tareas para prestar un servicio; tiempos de } \\
\text { proceso/suministro determinísticos y estocásticos; costos de prestación de servicios. }\end{array}$ \\
\hline $\begin{array}{l}\text { ¿El problema refleja } \\
\text { la complejidad de los } \\
\text { problemas de la vida } \\
\text { real? }\end{array}$ & $\begin{array}{l}\text { La complejidad del proceso contratación e integración de personal se da en tres } \\
\text { dimensiones: } \\
\text { a) Comportamiento aleatorio en tiempos de duración de las tareas } \\
\text { b) Alta interdependencia entre las etapas del proceso (ver figura 2) } \\
\text { c) Necesidad de estudiar el comportamiento del proceso durante un periodo de tiempo } \\
\text { para resolver el problema (lo que demora tramitar 100 contrataciones de personal) bajo } \\
\text { ciertos valores de las variables de decisión }\end{array}$ \\
\hline
\end{tabular}


En la figura 9 se advierte que de 100 instancias de proceso que inician, 13 se encaminan por las rutas alternas; de las cuales 6 de ellas suspenden su flujo por no aceptar las políticas de la compañía y las otras 7 por no entregar a tiempo la documentación faltante. El indicador en la parte superior derecha de cada actividad señala el número de instancias completadas. Al ejecutar la simulación en el nivel de análisis de tiempo, se obtienen los resultados mostrados en la Tabla 2.

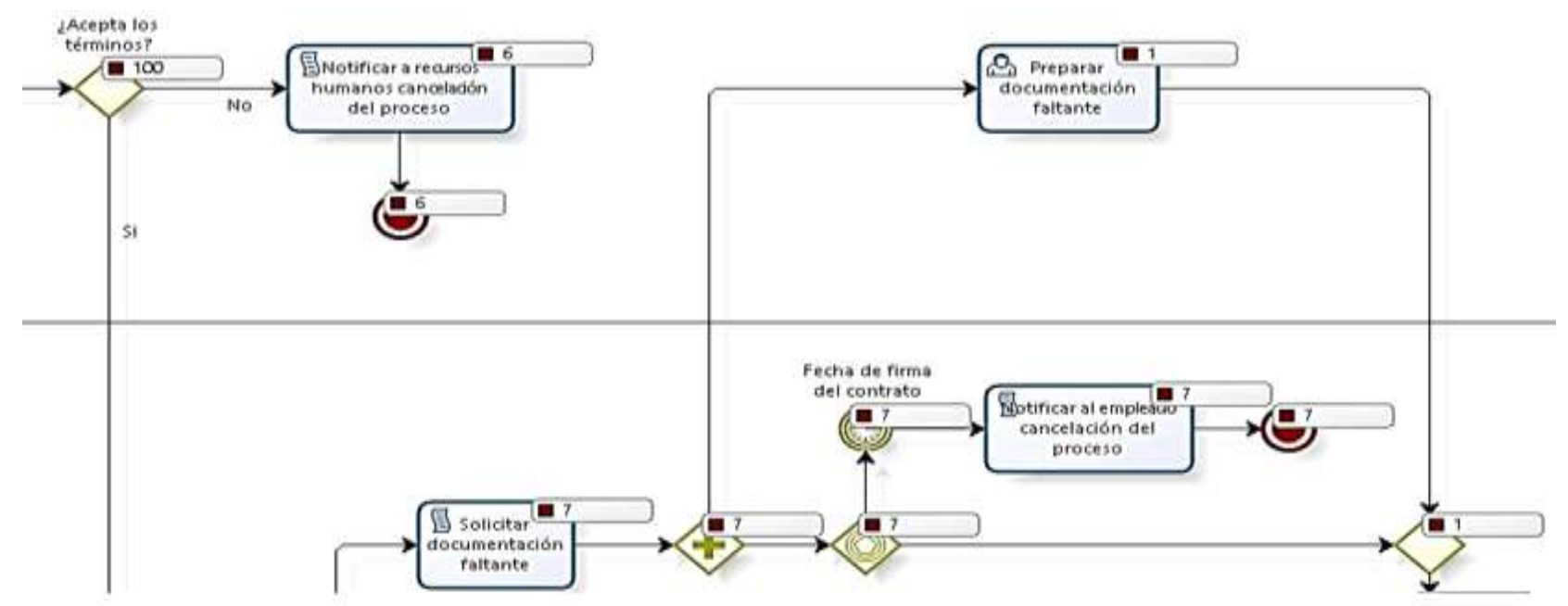

Fig. 9. Vista parcial de entradas/salidas simuladas en cada tarea/compuerta del proceso.

Tabla 2: Tiempos requeridos para procesar 100 posibles contrataciones de empleados en días (d), horas (h), minutos (m)

\begin{tabular}{|c|c|c|c|c|c|c|c|}
\hline Nombre & Tipo & $\begin{array}{l}\text { Instancias } \\
\text { completadas }\end{array}$ & $\begin{array}{l}\text { Instancias } \\
\text { iniciadas }\end{array}$ & $\begin{array}{l}\text { Tiempo } \\
\text { mínimo }\end{array}$ & $\begin{array}{l}\text { Tiempo } \\
\text { máximo }\end{array}$ & $\begin{array}{l}\text { Tiempo } \\
\text { promedio }\end{array}$ & $\begin{array}{l}\text { Tiempo } \\
\text { total }\end{array}$ \\
\hline $\begin{array}{l}\text { Contratación e Integración de } \\
\text { Personal }\end{array}$ & Proceso & 82 & 100 & $\begin{array}{l}\text { 00d } 11 \mathrm{~h} \\
38 \mathrm{~m}\end{array}$ & $\begin{array}{l}\text { 08d 00h } \\
48 \mathrm{~m}\end{array}$ & $\begin{array}{l}\text { 04d 20h } \\
04 \mathrm{~m}\end{array}$ & $\begin{array}{l}22 \mathrm{~d} 22 \mathrm{~h} \\
05 \mathrm{~m}\end{array}$ \\
\hline NoneStart & $\begin{array}{l}\text { Evento de } \\
\text { inicio }\end{array}$ & 100 & & & & & \\
\hline $\begin{array}{l}\text { Preparar documentos para la } \\
\text { contratación }\end{array}$ & Tarea & 90 & 90 & $\begin{array}{l}\text { 00d 02h } \\
17 \mathrm{~m}\end{array}$ & $\begin{array}{l}\text { 01d 04h } \\
05 \mathrm{~m}\end{array}$ & $\begin{array}{l}\text { 00d 08h } \\
36 \mathrm{~m}\end{array}$ & $\begin{array}{l}\text { 01d 07h } \\
00 \mathrm{~m}\end{array}$ \\
\hline $\begin{array}{l}\text { Solicitar permisos de } \\
\text { accesibilidad }\end{array}$ & Tarea & 98 & 98 & $\begin{array}{l}\text { 00d 00h } \\
05 \mathrm{~m}\end{array}$ & $\begin{array}{l}00 \mathrm{~d} 04 \mathrm{~h} \\
46 \mathrm{~m}\end{array}$ & $\begin{array}{l}00 \mathrm{~d} 00 \mathrm{~h} \\
36 \mathrm{~m}\end{array}$ & $\begin{array}{l}02 \mathrm{~d} 12 \mathrm{~h} \\
12 \mathrm{~m}\end{array}$ \\
\hline $\begin{array}{l}\text { Actividades previas al ingreso } \\
\text { del empleado }\end{array}$ & Compuerta & 100 & 100 & & & & \\
\hline Alistar Puesto de Trabajo & Tarea & 94 & 95 & $\begin{array}{l}\text { 00d 00h } \\
58 \mathrm{~m}\end{array}$ & $\begin{array}{l}00 \mathrm{~d} 23 \mathrm{~h} \\
13 \mathrm{~m}\end{array}$ & $\begin{array}{l}\text { 00d 05h } \\
32 \mathrm{~m}\end{array}$ & $\begin{array}{l}21 \mathrm{~d} 23 \mathrm{~h} \\
11 \mathrm{~m}\end{array}$ \\
\hline Fecha de firma del contrato & $\begin{array}{l}\text { Evento } \\
\text { intermedio }\end{array}$ & 82 & 82 & & & & \\
\hline Firmar Contrato & Tarea & 82 & 82 & $\begin{array}{l}\text { 00d 02h } \\
00 \mathrm{~m}\end{array}$ & $\begin{array}{l}04 d 22 h \\
17 m\end{array}$ & $\begin{array}{l}\text { 01d 15h } \\
38 \mathrm{~m}\end{array}$ & $\begin{array}{l}14 \mathrm{~d} 10 \mathrm{~h} \\
04 \mathrm{~m}\end{array}$ \\
\hline ParallelGateway & Compuerta & 82 & 94 & & & & \\
\hline NoneEnd & Evento de Fin & 82 & & & & & \\
\hline $\begin{array}{l}\text { Completar Actividades de la } \\
\text { primera semana }\end{array}$ & Tarea & 82 & 82 & $\begin{array}{l}\text { 00d 15h } \\
39 \mathrm{~m}\end{array}$ & $\begin{array}{l}\text { 02d 19h } \\
53 \mathrm{~m}\end{array}$ & $\begin{array}{l}01 \mathrm{~d} \mathrm{04h} \\
17 \mathrm{~m} \mathrm{32s}\end{array}$ & $\begin{array}{l}\text { 05d 15h } \\
57 \mathrm{~m}\end{array}$ \\
\hline $\begin{array}{l}\text { Fecha de Ingreso Nuevo } \\
\text { Empleado }\end{array}$ & $\begin{array}{l}\text { Evento } \\
\text { intermedio }\end{array}$ & 82 & 82 & & & & \\
\hline $\begin{array}{l}\text { Ingresar la información del } \\
\text { empleado seleccionado }\end{array}$ & Tarea & 100 & 100 & $\begin{array}{l}\text { 00d 00h } \\
06 \mathrm{~m}\end{array}$ & $\begin{array}{l}\text { 00d } 14 \mathrm{~h} \\
27 \mathrm{~m}\end{array}$ & $\begin{array}{l}\text { 00d } 02 \mathrm{~h} \\
37 \mathrm{~m}\end{array}$ & $\begin{array}{l}10 \mathrm{~d} 22 \mathrm{~h} \\
52 \mathrm{~m}\end{array}$ \\
\hline $\begin{array}{l}\text { Gestionar Creación de Usuario } \\
\text { y Permisos }\end{array}$ & Tarea & 95 & 98 & $\begin{array}{l}\text { 00d 00h } \\
59 \mathrm{~m}\end{array}$ & $\begin{array}{l}01 \mathrm{~d} 10 \mathrm{~h} \\
47 \mathrm{~m}\end{array}$ & $\begin{array}{l}\text { 00d 09h } \\
20 \mathrm{~m}\end{array}$ & $\begin{array}{l}07 d 02 h \\
54 m\end{array}$ \\
\hline $\begin{array}{l}\text { Solicitar documentación } \\
\text { faltante }\end{array}$ & Tarea & 8 & 8 & $\begin{array}{l}\text { 00d 00h } \\
03 \mathrm{~m}\end{array}$ & $\begin{array}{l}\text { 00d 13h } \\
45 \mathrm{~m}\end{array}$ & $\begin{array}{l}\text { 00d 02h } \\
50 \mathrm{~m}\end{array}$ & $\begin{array}{l}00 \mathrm{~d} 22 \mathrm{~h} \\
47 \mathrm{~m}\end{array}$ \\
\hline Enviar Plan de Actividades & Tarea & 95 & 95 & $\begin{array}{l}\text { 00d 00h } \\
01 \mathrm{~m}\end{array}$ & $\begin{array}{l}\text { 00d 07h } \\
31 \mathrm{~m}\end{array}$ & $\begin{array}{l}\text { 00d 00h } \\
28 \mathrm{~m}\end{array}$ & $\begin{array}{l}01 \mathrm{~d} 20 \mathrm{~h} \\
47 \mathrm{~m}\end{array}$ \\
\hline ¿Hacen falta documentos? & Compuerta & 90 & 90 & & & & \\
\hline
\end{tabular}


Tabla 2 (continuación)

\begin{tabular}{|c|c|c|c|c|c|c|c|}
\hline Nombre & Tipo & $\begin{array}{l}\text { Instancias } \\
\text { completadas }\end{array}$ & $\begin{array}{l}\text { Instancias } \\
\text { iniciadas }\end{array}$ & $\begin{array}{l}\text { Tiempo } \\
\text { mínimo }\end{array}$ & $\begin{array}{l}\text { Tiempo } \\
\text { máximo }\end{array}$ & $\begin{array}{l}\text { Tiempo } \\
\text { promedio }\end{array}$ & $\begin{array}{l}\text { Tiempo } \\
\text { total }\end{array}$ \\
\hline $\begin{array}{l}\text { Enviar reglamento de trabajo y } \\
\text { otras políticas }\end{array}$ & Tarea & 100 & 100 & $\begin{array}{l}\text { 00d 00h } \\
01 \mathrm{~m}\end{array}$ & $\begin{array}{l}\text { 00d 13h } \\
10 \mathrm{~m}\end{array}$ & $\begin{array}{l}\text { 00d 00h } \\
29 \mathrm{~m}\end{array}$ & $\begin{array}{l}\text { 02d } \\
00 \mathrm{~h} \\
37 \mathrm{~m}\end{array}$ \\
\hline EventBasedGateway & Compuerta & 8 & 8 & & & & \\
\hline Fecha de firma del contrato & $\begin{array}{l}\text { Evento } \\
\text { intermedio }\end{array}$ & 8 & 8 & & & & \\
\hline $\begin{array}{l}\text { Notificar al empleado } \\
\text { cancelación del proceso }\end{array}$ & Tarea & 8 & 8 & $\begin{array}{l}\text { 00d 00h } \\
01 \mathrm{~m}\end{array}$ & $\begin{array}{l}\text { 00d 00h } \\
02 \mathrm{~m}\end{array}$ & $\begin{array}{l}\text { 00d 00h } \\
01 \mathrm{~m}\end{array}$ & $\begin{array}{l}00 \mathrm{~d} \\
00 \mathrm{~h} \\
10 \mathrm{~m}\end{array}$ \\
\hline TerminateEnd & $\begin{array}{l}\text { Evento de } \\
\text { Fin }\end{array}$ & 8 & & & & & \\
\hline ExclusiveGateway & Compuerta & 82 & 82 & & & & \\
\hline Elaborar plan de actividades & Tarea & 98 & 100 & $\begin{array}{l}\text { 00d 01h } \\
00 \mathrm{~m}\end{array}$ & $\begin{array}{l}01 d 16 h \\
32 m\end{array}$ & $\begin{array}{l}\text { 00d 06h } \\
47 \mathrm{~m}\end{array}$ & $\begin{array}{l}28 d \\
07 h \\
26 m\end{array}$ \\
\hline ¿Acepta los términos? & Compuerta & 100 & 100 & & & & \\
\hline $\begin{array}{l}\text { Leer reglamento y demás } \\
\text { políticas }\end{array}$ & Tarea & 100 & 100 & $\begin{array}{l}\text { 00d 03h } \\
34 \mathrm{~m}\end{array}$ & $\begin{array}{l}02 d 20 h \\
34 m\end{array}$ & $\begin{array}{l}\text { 01d 01h } \\
07 \mathrm{~m}\end{array}$ & $\begin{array}{l}13 \mathrm{~d} \\
17 \mathrm{~h} \\
16 \mathrm{~m} \\
\end{array}$ \\
\hline $\begin{array}{l}\text { Notificar a recursos humanos } \\
\text { cancelación del proceso }\end{array}$ & Tarea & 10 & 10 & $\begin{array}{l}\text { 00d 00h } \\
01 \mathrm{~m}\end{array}$ & $\begin{array}{l}01 d 06 h \\
22 m\end{array}$ & $\begin{array}{l}\text { 00d 05h } \\
37 \mathrm{~m}\end{array}$ & $\begin{array}{l}02 d \\
08 \mathrm{~h} \\
11 \mathrm{~m} \\
\end{array}$ \\
\hline TerminateEnd & $\begin{array}{l}\text { Evento de } \\
\text { Fin }\end{array}$ & 10 & & & & & \\
\hline ParallelGateway & Compuerta & 8 & 8 & & & & \\
\hline Recepción de documentos & $\begin{array}{l}\text { Evento } \\
\text { intermedio }\end{array}$ & 0 & 1 & & & & \\
\hline Preparar documentación faltante & Tarea & 1 & 1 & $\begin{array}{l}\text { 00d 00h } \\
01 \mathrm{~m}\end{array}$ & $\begin{array}{l}\text { 00d } 18 \mathrm{~h} \\
27 \mathrm{~m}\end{array}$ & $\begin{array}{l}\text { 00d 02h } \\
19 \mathrm{~m}\end{array}$ & $\begin{array}{l}00 \mathrm{~d} \\
18 \mathrm{~h} \\
36 \mathrm{~m}\end{array}$ \\
\hline ExclusiveGateway & Compuerta & 1 & 1 & & & & \\
\hline
\end{tabular}

En la Tabla 1 puede verse la duración mínima, promedio y máxima de cada una de las tareas en la simulación, así como su tiempo total. Obsérvese, por ejemplo, que en promedio la contratación de un empleado puede tardarse $04 \mathrm{~d} 20 \mathrm{~h} 4 \mathrm{~m}$ en ser procesada, teniendo en cuenta la jornada laboral de 8 horas por día, determinada en el análisis de calendarios. Igualmente el tiempo total requerido para procesar hasta 100 posibles contrataciones de personal es de $22 \mathrm{~d} 22 \mathrm{~h} 05 \mathrm{~m}$. Estos valores se obtuvieron de acuerdo a los parámetros ingresados en el programa, los cuales pueden ser modificados por el usuario (docenteestudiante) y cuyo juego de valores de decisión puede considerarse un posible problema a solucionar en el contexto de ABP (Vizcarro y Juarez, 2008), según se describe en la Tabla 1.

En relación con los recursos y calendarios, al ejecutar la simulación se presenta, a modo de ventana emergente, los porcentajes de utilización de cada uno de los recursos. Estos valores varían constantemente a lo largo de la simulación, pero en todo momento el nuevo empleado es quien tiene el más alto grado de uso, tal como se muestra en la Tabla 3. Esta observación es consecuente con la noción de que el empleado a contratar es quien determina el ritmo del proceso, pues las actividades que él realiza son las que pueden presentar mayor variabilidad respecto a las tareas de los entes de la organización.

Tabla 3: Tasa de utilización de recursos

\begin{tabular}{|l|r|}
\hline Recurso & \multicolumn{1}{l|}{$\begin{array}{l}\text { Uso } \\
(\%)\end{array}$} \\
\hline Jefe del nuevo empleado & 7,75 \\
\hline Analista de talento humano & 6,88 \\
\hline Nuevo empleado & 16,78 \\
\hline $\begin{array}{l}\text { Administrador área } \\
\text { tecnológica }\end{array}$ & 3,82 \\
\hline
\end{tabular}


Finalmente se realizó una prueba piloto. Esta se aplicó a diecinueve estudiantes de ingeniería industrial del curso de gestión humana del primer semestre de 2015 con el acompañamiento del respectivo docente. La prueba consistió en que el docente explicara el proceso de contratación e integración de personal apoyándose en la herramienta informática BPSim descrita, empleando la instancia de problema mostrado en la Tabla 1 y partiendo de ello, se le realizaron a los estudiante cuatro preguntas relacionadas para que respondieran en una escala de 1 a 5 (1 totalmente en desacuerdo, 2 en descuerdo, 3 neutral, 4 de acuerdo calificación y 5 totalmente de acuerdo). En la Tabla 4, se muestran las respuestas a c/u de las preguntas.

Tabla 4: Resultados de aplicación de la prueba piloto

\begin{tabular}{|c|c|c|c|c|c|}
\hline Pregunta/Calificación & 1 & 2 & 3 & 4 & 5 \\
\hline $\begin{array}{l}\text { ¿Considera que la herramienta presentada ilustra claramente el } \\
\text { proceso de contratación de personal? }\end{array}$ & & & $16 \%$ & $37 \%$ & $47 \%$ \\
\hline ¿Comprendió fácilmente el funcionamiento de la herramienta? & & & $16 \%$ & $32 \%$ & $53 \%$ \\
\hline $\begin{array}{l}\text { ¿La herramienta le ha permitido complementar sus conocimientos } \\
\text { sobre el proceso de contratación de personal? }\end{array}$ & & & $11 \%$ & $63 \%$ & $26 \%$ \\
\hline $\begin{array}{l}\text { ¿Cree que la herramienta sería útil como apoyo didáctico para el } \\
\text { docente en los procesos de enseñanza-aprendizaje? }\end{array}$ & & & & $37 \%$ & $63 \%$ \\
\hline
\end{tabular}

Al momento de realizar la prueba, se comprobó que para comprender el proceso en BPSIM los estudiantes debían tener nociones básicas sobre la notación en BPM. Es de connotar que respecto a la pregunta ¿Considera que la herramienta presentada ilustra claramente el proceso de contratación de personal? El 37\% considero estar de acuerdo y el $47 \%$ totalmente de acuerdo. Igualmente respecto a la pregunta ¿Cree que la herramienta sería útil como apoyo didáctico para el docente en los procesos de enseñanza-aprendizaje? El 37\% considero estar de acuerdo y el $63 \%$ totalmente de acuerdo, lo cual es un indicativo de la aceptación de BPSIM como soporte didáctico en el aprendizaje de la gestión de procesos de servicio. En general, se percibió durante la realización de la prueba piloto que entre los atributos del modelo que más llamaron la atención están: la interfaz visual, la interactividad del modelo que permite modificar datos como tiempos o recursos que operan (variables de decisión), la dinámica que se observa en el modo de simulación y la representación de los actores que intervienen en el proceso. Cabe destacar, además, que la metodología BPSIM es poco conocida en el medio educativo local, específicamente en programas de ingeniería industrial (quizás por lo nueva), pues los estudiantes encuestados coincidieron en que no conocían este tipo de simulación, pero que les gustaría que fuera aplicada en otras áreas objeto de estudio de su carrera.

\section{CONCLUSIONES}

De los resultados y el análisis expuesto se pueden obtener las siguientes conclusiones:

La tecnología BPSIM permitió fácilmente la modelación del proceso de servicio contratación e integración de personal y su simulación, propiciando así un entorno de aprendizaje accesible, amigable con el estudiante e interactivo, pues las variables de decisión, como se señala en la discusión de los resultados, pueden ser modificadas por el usuario del modelo en la medida en que lo considere pertinente.

Mediante la simulación del proceso de contratación e integración de personal modelado con BPMN, es posible a cero costos, entrenar a futuros ingenieros industriales en la predicción del desempeño de un sistema de servicios. Este desempeño puede medirse tanto desde el punto de vista del nivel de servicio al cliente, como desde el punto de vista del administrador del sistema, de acuerdo al uso de los recursos.

\section{AGRADECIMIENTOS}

Los autores agradecen a la Universidad Nacional de Colombia sede Manizales a través de la dirección de investigaciones (DIMA) y Colciencias por el apoyo institucional y financiero al desarrollo de esta investigación (proyecto: " Semillero de investigación en simulación de sistemas de producción y de servicios. Aplicaciones didácticas " código Hermes 21854).

\section{REFERENCIAS}

Barjis, J., Verbraeck, A., Enterprise modelling and simulation in a broader context. International Journal of Simulation \& Process Modelling. Volume 4, Nos. 3/4, (2008) 
Bizagi, [en línea] Bizagi BPM Suite Functional Description,

https://www.bizagi.com/docs/BizAgi\%20Functional\%20Description.pdf. Acceso: 17 noviembre (2014)

Bolaños, S. J. y López, S. A. LMPS como propuesta alterna a BPMN para el modelado de procesos de software. Tecnura. Bogotá : s.n., Vol. 16, 34 (2012). Disponible en

http://www.scielo.org.co/scielo.php?script=sci_arttext\&pid=S0123-921X2012000400012\&lang=es. ISSN 0123-921X.

Casadei C., L., La simulación como herramienta de aprendizaje en física. Actualidades Investigativas en Educación. [online]. Vol. 8, 2, págs. 1-27 (2008). Disponible en

http://revista.inie.ucr.ac.cr/uploads/tx_magazine/fisica_01.pdf. ISSN 1409-4703

Espinosa, Y., López, C. R. Business Process Modeling: Evolution of the Concept in a University Context. Computación y Sistemas. Vol. 17, 1, págs. 79-93 (2013.). Disponible en http://www.scielo.org.mx/scielo.php?script=sci_arttext\&pid=S1405-55462013000100009\&lang=es.

Giraldo, J. A., Toro, C. A., Jaramillo, F. A., Aprendiendo sobre la Secuenciación de Trabajos en un Job Shop mediante el Uso de Simulación. Form. Univ., La Serena, v. 6, n. 4 (2013)

González G., O. E., Patarroyo D., N. I. Competencias específicas solicitadas al recién egresado de Ingeniería Industrial por el sector servicios en Bogotá. Ciencia e Ingeniería Neogranadina. [online]. Vol. 24, 1 (2014). Disponible en http://www.scielo.org.co/scielo.php?script=sci_arttext\&pid=S012481702014000100009 \&lang=es.

IBM, [en línea] The INNOV8 BPM simulation game brings IT and business together for process model innovation, http://www-01.ibm.com/software/solutions/soa/innov8/index.html. Acceso: 17 enero (2015).

Márquez G., C. V., y otros. Satisfacción académica con el abp en estudiantes de literatura de la Universidad de Colima, Mexico. Revista Intercontinental de Psicología y Educación. Vol. 13, 1, págs. $29-44$ (2011). Disponible en: http://www.redalyc.org/articulo.oa?id=80218382003. ISSN 0187-7690.

Melao, N. y Pidd, M. Use of Business Process Simulation: A Survey of Practitioners. The Journal of the Operational Research Society. Vol. 54, 1, págs. 2-10 (2003). Disponible en

http://www.jstor.org/stable/822743. ISSN 0160-5682.

Morales G., M. L., Empleo del aprendizaje basado en problemas (abp). Una propuesta para acercarse a la química verde. Tecnología en Marcha. s.l. : Editorial Tecnológica de Costa Rica, Vol. 21, 1, págs. 41-48 (2008). Disponible en http://tecdigital.tec.ac.cr/servicios/ojs/index.php/tec_marcha/article/view/1337/1239.

Oracle, [en línea] Running Simulations in Oracle BPM, http://docs.oracle.com/cd/E17904_01/doc.1111/e15176/simul_bpmpd.htm\#BPMPD1619 Acceso: 17 enero (2015)

Riascos, S. C., Ávila, G. P., Quintero, D. M., Las TIC en el aula: percepciones de los profesores universitarios. Educación y Educadores. [online]. Vol. 12, 3 (2009). Disponible en http://www.scielo.org.co/scielo.php?script=sci_arttext\&pid=S0123-12942009000300008. ISSN 0123-1294.

Suarez, R., La Educación, 1르 edición, Editorial Trillas S.A. México, D.F. (1985)

Ventura, A. C., Estilos de aprendizaje y prácticas de enseñanza en la universidad. Un binomio que sustenta la calidad educativa. Perfiles educativos: 33(esp), (2011)

Vivas, N. A., Estrategias de aprendizaje. Góndola. [online]. Vol. 5, 1, págs. 27-37, (2010). Disponible en http://revistas.udistrital.edu.co/ojs/index.php/GDLA/article/viewFile/5220/6850.

Vizcarro C. y Juárez E., ¿Qué es y cómo funciona el aprendizaje basado en problemas?, en La Metodología del Aprendizaje Basado en Problemas, Murcia, España (2008) 